Gewaltenteilung an. Doeker vergißt auch nicht, die Wahlverfahren darzustellen, sowie die Strukturen und Verfahren in den Parteien, insbesondere bei der Wahlvorbereitung und der Kandidatenaufstellung. Soweit erforderlich, sind wichtige und seltene Dokumente in den Text eingearbeitet, so etwa aus Indien die bemerkenswerte Anleitung zur Benotung der Abgeordneten am Ende einer Legislaturperiode. Es fehlt auch nicht ein Abriß der Geschichte der wichtigsten Parteien und eine knappe Soziologie der Parlamente. Bei den Darstellungen des Wahlverfahrens wird sehr anschaulich, welche gewaltige administrative Leistung ein Land wie Indien erbringt, wenn dort eine Wahl durchgeführt wird. Der Einsatz und die Opfer eines solchen Verfahrens belegen mehr als große Worte eine feste Verankerung der Demokratie in der führenden Schicht des Landes. Doeker vergißt bei seiner Darstellung auch nicht die Wahlkreiseinteilung und die Reaktion der Wähler auf die Wahlverfahren. Diese Detailfreude ist dankenswert. Wenn dies erst der einführende Teil sein soll, darf man auf den Hauptband gespannt sein.

Für den zweiten Band wünschte ich mir allerdings eine sorgfältigere Redaktion und Korrektur durch Autor und Lektor. Daß Herbert Krüger als Horst Krüger zitiert wird (S. 6 Anm. 12), muß diese Zeitschrift natürlich übelnehmen. Aber einen Satz wie: "Die Nichtausübung des Wahlrechts in Australien hat zu einer durchschnittlichen Wahlbeteiligung um 95-96\% geführt." (S. 254) - gemeint war nach dem Zusammenhang die gesetzliche Wahlpflicht in Australien - sollte der Verfasser sich selbst sehr übelnehmen. Derartiges trübt die Freude über ein interessantes Buch.

Dieter Schröder

\title{
Zehra Önder
}

Saudi-Arabien. Zwischen islamischer Ideologie und westlicher Okonomie Klett-Cotta, Stuttgart 1980, 405 S., DM 78,-

Mit der vorliegenden Arbeit hat die Verfasserin einen sehr wichtigen Beitrag zur Erforschung der Innen- und Außenpolitik Saudi-Arabiens geleistet und damit eine oft als schmerzlich empfundene Forschungslücke geschlossen. In mancher Hinsicht etwas anders angelegt als das epochale Werk von Haas über Jordanien, kommt Onders Buch als erstem deutschsprachigen seiner Art über das wahhabitische Königreich für die Politikwissenschaft und Orientalistik hierzulande doch die gleiche Bedeutung zu. Der Autorin ist zudem gelungen, was man heutzutage leider nur recht selten konstatieren kann: die Verbindung von Wissenschaftlichkeit und Verständlichkeit auch für den interessierten Nichtfachmann. Dabei werden viele Einzelheiten vermittelt, ohne daß die großen Zusammenhänge verlorengingen, was bei einem so komplexen Thema wie diesem zweifellos nicht einfach ist. In einer Zeit, in der vorwiegend Spezialstudien, deren Relevanz für die Forschung nicht in jedem Fall gleich auf den ersten Blick einleuchtet, produziert werden, muß eine Gesamtdarstellung ohnehin sehr begrüßt werden. 
Am Anfang der übersichtlich gegliederten Arbeit steht ein Kapitel über den Wandel der saudiarabischen Außenpolitik vom begrenzten Isolationismus in der Zeit der Staatsgründung zur aktiven islamorientierten Politik zu Beginn der siebziger Jahre. Daran schließt sich eine Analyse der Entscheidungsträger (der von Onder benutzte Ausdruck "Entscheidungsmechanismen" ist nicht ganz glücklich gewählt) an, die neben einer Untersuchung der politischen Elite (Stammesführer, religiöse Oligarchie, Minister und sonstige westlich ausgebildete Berater) auch eine der "Royal Family" umfaßt. Den größten Raum nimmt das darauf folgende Kapitel über den ideologisch-kulturellen Orientierungsrahmen ein. Hier werden Pan-Arabismus, Pan-Afrikanismus und Pan-Islamismus ausführlich und sehr kenntnisreich abgehandelt, wobei die zentrale Bedeutung Israels und der Palästina-Frage klar herausgearbeitet wird. Allerdings muß in Bezug auf das israelisch-arabische Verhältnis auf die einzige, nach Ansicht des Rezensenten aber nicht gravierende Schwäche dieses ansonsten so ausgezeichneten Buches hingewiesen und angemerkt werden, daß die Verfasserin zwar die Sicht Riads deutlich werden läßt, aber die kritische Distanz zur saudischen Argumentation und Betrachtungsweise häufig nicht genug gewahrt wird. Der Rezensent gesteht gerne zu, daß manches verschieden interpretierbar ist, doch sehr realistisch scheint ihm schon in Anbetracht der militärischen Möglichkeiten z. B. die Unterstellung, daß Israels Expansionsgelüste selbst vor weiten Teilen Saudi-Arabiens nicht haltmachen würden (S. 128, ähnlich S. 130), nicht zu sein. (Die Autorin stützt sich dabei zudem z. T. auf mißverstandene oder überinterpretierte Quellenpassagen und z. T. auf fragwürdige Informationsgrundlagen.) Auch ist es zumindest erklärungsbedürftig, wenn man von einer "aggressiven" Politik des Judenstaates spricht (ebenda), denn es fragt sich, ob man mit einer solchen Formulierung der verwickelten Situation im Nahen Osten und dem Präventivkriegsproblem 1956 und 1967 gerecht wird. Da aber nicht nur das Sein, sondern auch der Schein das Bewußtsein bestimmt, ist es zweifellos von großer Bedeutung, zunächst einmal die - sicher subjektive - saudische Position vermittelt zu bekommen. Und dies geschieht auf der Grundlage neuen Quellenmaterials in ungewohnter Eindringlichkeit.

An das dritte Kapitel schließt sich eine Untersuchung der sicherheitspolitischen Orientierung an. Neben dem Verhältnis zu den Supermächten wird darin auch die Lage im Roten Meer und im Arabischen (Persischen) Golf erörtert. Dabei finden, was in der Natur der Sache liegt, die Vereinigten Emirate, der Oman und Äthiopien besondere Beachtung, ohne daß andere Aspekte des Problemkreises (z. B. Dschibuti) vernachlässigt werden. Politische Veränderungen in den beiden genannten Regionen können vielfältige Auswirkungen auf Saudi-Arabien haben; sie reichen von einer Bedrohung der für das Land lebenswichtigen Tanker-Routen bis zu der Ausstrahlung erfolgreicher sozialrevolutionärer oder religiös motivierter Bewegungen auf die gesamte Arabische Halbinsel. Die hier vermittelten Informationen dürften auch für Nicht-Arabisten und Nicht-Orientalisten von großem Interesse sein.

Im letzten Kapitel wird die wirtschaftspolitische Orientierung Riads behandelt. Dabei nimmt, was nicht weiter verwundern kann, das Thema "Erdöl und Industrialisierung" den größten Raum (45 Seiten) ein. (Die Problemkreise Landwirtschaft, Mittelstandspo- 
litik, Technologie-Tranfer und internationale Finanzpolitik fallen ebenfalls unter dieses große Thema, das seit den Ereignissen in Persien wieder mehr Beachtung gefunden hat.) Relativ knapp, aber instruktiv nimmt sich dagegen der darauf folgende Abschnitt "Dritte Welt" aus; den Abschnitt "Perspektiven" wünschte man sich vielleicht etwas umfangreicher.

Eine Reihe von Graphiken und Tabellen, zwei Karten, eine umfangreiche Bibliographie (28 Seiten) und ein Register erhöhen die Nützlichkeit des Werkes. Das Quellen- und Literaturverzeichnis sowie der Anmerkungsapparat machen deutlich, welche enormen Materialmengen (u. a. auch Rundfunk- und Fernsehsendungen, die im Fall eines so abgeschlossenen Landes wie Saudi-Arabien eine nicht zu unterschätzende Informationsquelle darstellen) die Autorin bewältigt hat. Einen besonderen Wert erhält die Arbeit durch die Auswertung von Interviews der Verfasserin mit 23 Politikern, Beamten und Publizisten, die dem islamischen Kulturkreis zuzurechnen sind. Darunter befinden sich zwölf hochrangige Gesprächspartner aus Saudi-Arabien. Man kann sich vorstellen, daß es gerade für eine Frau nicht einfach war, überhaupt empfangen zu werden, und es muß bezweifelt werden, daß ein Politologe in nächster Zukunft wieder Gelegenheit erhalten wird, derart viele Interviews mit so hochgestellten Persönlichkeiten des Landes durchzuführen. Hinzu kommt, daß auch eine Reihe von unveröffentlichten Dokumenten, wie z. B. die Manuskripte von Reden, die Onder zur Verfügung standen, aller Voraussicht nach auf Jahrzehnte der Forschung nicht zugänglich sein werden.

Nach Ansicht der Verfasserin ist eine Rückbesinnung auf die islamische Kultur erforderlich, um die ideologische Verwirrung der moslemischen Eliten zu überwinden. Das bedeute jedoch auch und gerade eine Lösung vom religiösen Formalismus und eine Orientierung an jener Periode des Islams, in der Kultur und Wissenschaft blühten, weil die Träger der Entwicklung zu schöpferischer Verarbeitung kritischer Anregungen und fremder Einflüsse fähig waren. Weder die sklavische Nachahmung westlicher Verhaltensweisen oder die einfache Ubernahme westlicher Normen und Technologien noch das unveränderte Reproduzieren der Verhältnisse aus längst vergangenen "guten alten Zeiten" einstiger Macht und Größe könne eine Lösung der gegenwärtigen Probleme darstellen, wie die Autorin überzeugend ausführt. Das eine hieße Verwestlichung ohne Vorhandensein der erforderlichen Grundlagen, das andere Stagnation, ja Rückschritt. Ansätze für eine den Erfordernissen der Gegenwart gerecht werdende, zukunftsweisende Interpretation des Korans seien durchaus vorhanden. Von entscheidender Bedeutung für die Uberwindung des augenblicklichen Vakuums der Werte ist nach Onders Meinung die Ausrichtung der Außenpolitik am Pan-Arabismus und Pan-Islamismus, verbunden mit einer entsprechenden, an den sozioökonomischen, kulturellen und historischen Gegebenheiten orientierten Innenpolitik. Saudi-Arabien als Zentrum des Islams und finanzwirtschaftliche Großmacht könnte Vorbild für andere Staaten des Kulturkreises werden und - was in gewissem Umfang bereits geschehe - dort die Entwicklung fördern. Riad könne dadurch einen bedeutenden Beitrag zu einem erfolgreichen Nord-Süd-Dialog leisten und seine Führungsposition im arabisch-islamischen Raum langfristig sichern. 
Das vorliegende Buch darf mit Fug und Recht als eine überaus gelungene Analyse der saudischen Innen- und Außenpolitik sowie der Möglichkeiten einer Renaissance des Islams bezeichnet werden. Auf absehbare Zeit dürfte es das Standardwerk über das wahhabitische Königreich sein und bleiben. Diese Feststellung bezieht sich ausdrücklich nicht nur auf die deutschsprachige Literatur! Politikwissenschaftler, Orientalisten und Arabisten werden an diese Arbeit auf lange Jahre hinaus ebensowenig vorbeigehen können wie der interessierte Laie.

Karl-Heinz Schmick

\section{Rudolf J. Lauff}

Die Außenpolitik Algeriens, 1962-1978;

Afrika Studien Nr. 107, IFO-Institut für Wirtschaftsforschung e. V. München, Weltforum Verlag, München-London, 1981, 227 S., DM 48,-

\section{Slimane Chikh}

\section{La politique af ricaine de l'Algérie}

Annuaire de l'Afrique du Nord, XVII, 1978, p. 1-54, Editions du Centre National de la Recherche Scientifique, Paris, 1979.

Das unabhängige Algerien fand in der deutschen Offentlichkeit und selbst in politischen Kreisen nur zurückhaltendes Interesse. Man war geneigt, dies Land trotz seines schweren und schließlich erfolgreichen Freiheitskampfes immer noch als "Jagdrevier" (chasse gardée) der Franzosen anzusehen. So gab es bei uns auch kaum Untersuchungen und Veröffentlichungen über die politischen, wirtschaftlichen, sozialen und kulturellen Entwicklungen Algeriens. Man war insoweit völlig auf die zahlreichen und gründlichen $\mathrm{Pu}-$ blikationen aus Frankreich angewiesen. Das vorliegende Buch von Rudolf J. Lauff über die Außenpolitik Algeriens 1962-1978 stößt daher bei uns in eine wirkliche Lücke und ist insoweit begrüßenswert. Der Verfasser hat damit eine große Aufgabe übernommen, die bei der Lebendigkeit und Vielfältigkeit der algerischen Außenpolitik und der Fülle des Materials nicht ganz leicht zu erfüllen war. Ihm dienten - wie er eingangs sagt - in erster Linie Zeitungsartikel als Quellengrundlage. Das aber erscheint wohl kaum ausreichend für eine sich als wissenschaftlich qualifizieren wollende Arbeit und führt, wie wir sehen werden, zu falschen oder schiefen Darstellungen. Der Verfasser hätte schon bei einem so komplexen Thema gründlicher recherchieren müssen, wozu sich in erster Linie die französischen Quellen anboten. Bedauerlich ist auch, daß diese Arbeit, die die Entwicklung bis 1978 erfaßt, erst drei Jahre später erscheint. Die in dem Werk wiedergegebenen Statistiken enden sogar schon mit 1975 oder teils noch früher. Dadurch fehlt es dem Buch leider an der wünschenswerten Aktualität. Andererseits stellt das Jahr 1978 mit dem Tode des bedeutenden Präsidenten Boumediène einen gewissen Abschluß in der 